\title{
Comparison of Maxillary Central Incisor Labial Crown-Root Angle with Collum Angle in Class I and Class II Division 2 Malocclusion
}

\author{
Subashree Rathi Selvan ${ }^{1}$, Saravana Pandiyan K.ㄹ, Ravindra Kumar Jain ${ }^{3}$ \\ ${ }^{1}$ Department of Orthodontics and Dentofacial Orthopaedics, Saveetha Dental College, SIMATS, Chennai, \\ Tamilnadu, India. ${ }^{2}$ Department of Orthodontics and Dentofacial Orthopaedics, Saveetha Dental College, \\ SIMATS, Chennai, Tamilnadu, India. ${ }^{3}$ Department of Orthodontics and Dentofacial Orthopaedics, Saveetha \\ Dental College, SIMATS, Chennai, Tamilnadu, India.
}

\section{ABSTRACT}

\section{BACKGROUND}

To anticipate difficulties with intrusion, extrusion, or torqueing mechanics in certain malocclusions, a thorough understanding of crown-root relationships in the buccolingual plane is warranted. This study aimed at examining the collum angle of the maxillary central incisor in Angle's class I and class II division 2 malocclusion and compare these values with the Labial Crown Root Angle (LCRA).

\section{METHODS}

This study was conducted on two groups i.e., group 1 comprising of 26 Angle's Class 1 subjects and group 2 comprising of 26 Class II div 2 subjects. Cephalometric measurements of collum angle and LCRA of maxillary central incisors were recorded for each subject and were compared between the two groups.

\section{RESULTS}

The mean Labial Crown-Root Angle was $28.35 \pm 4.01$ and $38.51 \pm 5.80$ for class I and class II division malocclusions respectively. Compared to the LCRA of Angle's class I malocclusion, the mean LCRA of Angle's class II division 2 was found to be greater, and is statistically significant. There was no significant gender variation. The mean difference between class I and class II div 2 malocclusion was found to be $9.42 \pm 2.23$ for collum angle and 10.16 \pm 1.79 in LCRA respectively.

\section{CONCLUSIONS}

The Labial Crown Root Angle and collum angle of Angle's Class II div 2 malocclusion were greater than the corresponding values in Angle's Class I malocclusion. Also, the LCRA is a more simplified method for measuring the crown root angle and can be used as an adjunct to collum angle.

\section{KEY WORDS}

Labial Crown-Root Angle (LCRA), Collum Angle, Maxillary Central Incisor, Class II Division 2
Corresponding Author: Dr. Subashree Rathi Selvan, Postgraduate Student, Department of Orthodontics \& Dentofacial Orthopaedics, Saveetha Dental College, SIMATS, Chennai-600077,

Tamilnadu, India.

E-mail: subashree.rathi@gmail.com

DOI: $10.14260 /$ jemds/2020/505

How to Cite This Article:

Selvan SR, Pandiyan SK, Jain RK. Comparison of maxillary central incisor labial crown-root angle with collum angle in class $I$ and class II division 2 malocclusion. J Evolution Med Dent Sc 2020;9(33):2324-2328, DOI: $10.14260 /$ jemds/2020/505

Submission 05-03-2020, Peer Review 07-07-2020, Acceptance 15-07-2020, Published 17-08-2020.

Copyright (C) 2020 JEMDS. This is an open access article distributed under Creative Commons Attribution License [Attribution 4.0 International (CC BY 4.0)] 


\section{BACKGROUND}

Since 1968, the modern orthodontic practice is largely based on the straight wire edgewise appliance, which was originally described by Andrews. The limitations of the straight-wire appliance become apparent when one considers the inherently variable natural crown forms, as well as the variability of root position in relation to the clinical crown.[1] The variability in natural crown forms is compensated by making alterations in the wire or bracket position. Variation of root position in relation to the crown is little spoken of in the clinical scenario.

Improvement in facial aesthetics is also one of the important motivating factors for patients to seek orthodontic treatment.[2] Smile plays a critical role in dental aesthetics and a person's social behaviour. ${ }^{[3][4]}$ The distinct morphology of maxillary central incisor is a key factor in achieving an aesthetic, functional, and stable Class I incisor relationship with orthodontic treatment.[5] The anteroposterior position of maxillary central incisor plays a key role in defining this aesthetics. [6]

Universally, it is accepted that the root angulation in relation to the crown is zero in most of the cases, despite the variations been described by various authors. ${ }^{[7][8] ~ T h e ~ C r o w n ~}$ to root angulation of maxillary central incisors may limit the degree to which the roots of these teeth can be torqued palatally when related to the maxillary palatal cortical plate of bone. [9] Collum angle of single rooted teeth is of particular interest to orthodontists as any variation in root angulations lends to unpredictable axial force application in movements. Varied root angulations confound intended axial loads on the tooth for intrusion and extrusion and the root may encroach the labial or lingual cortical plate when repositioned.[5]

Various studies indicate that the relationship of root to that of crown is variable in different types of malocclusion. It was also found by Delivanis and Kuftinecthat in Class II, Division 2 patients that the crowns of the maxillary central incisors tends to be "bent" to the lingual more often than in patients with other types of malocclusion. ${ }^{[8]}$ It was also said by the authors that in a Class II division 2 case, the crown root angulation may affect the orthodontic intrusion as well as the torque. In severe cases, this angulation variation may also increase the danger of perforating the palatal cortical plate. It has been postulated by Backlund that the crown root angulation to be a contributing factor in the development of Class II, Division 2 malocclusions.[10]

Most frequently, Collum angle has been investigated using lateral cephalometric radiographs. CT (Computed Tomography) and CBCT (Cone Beam Computed Tomography) have also been used alternatively in the recent times. But, cephalometrics is still considered to be satisfactory.

To achieve greater predictability in root position, and to anticipate difficulties with intrusion, extrusion, or torqueing mechanics, a thorough understanding of crown-root relationships in the bucco-lingual plane, and their application to clinical practice, is warranted. The purpose of this study was aimed at measuring the mean Collum Angle of maxillary central incisor in Angle's class I and Angle's Class II division 2 malocclusion. This value is also compared with the labial crown root angle (LCRA) of the same teeth and gender variation is studied.

\section{METHODS}

The study included lateral cephalograms of patients in the department of a private dental college in Chennai. A total of 52 cephalograms were studied of which 25 were male and 27 were female subjects. The study was categorized into two groups based on the type of malocclusion using Angle's classification of malocclusion: class I malocclusion and class II division 2 malocclusion.

\section{Inclusion Criteria}

- Group 1 included sample with normal occlusion designated as Angle's class I molar relationship.

- $\quad$ Group 2 included samples of Angle's class II division 2 malocclusion.

\section{Exclusion Criteria}

- Subjects that had orthodontic treatment earlier were excluded from the study.

- $\quad$ Subjects whose cephalometric radiographs were of poor quality or those for whom measurements were not easily readable were excluded.

- The digitized cephalometric radiographs were loaded into the Facad software (a software used for standard cephalometric tracing) for landmark identification and measurement. A customised analysis was then created to serve the needs for this study.

\section{Measurement of Collum Angle (CA)}

The Collum Angle (CA) is traditionally measured according to three points on the most anterior maxillary central incisor: the undamaged incisal edge (incisor superius, or IS), the constructed bisection of the facial and lingual cementoenamel junctions (fCEJ and ICEJ, respectively), and the anatomic root apex (upper incisor apicale).[11] The superius point of the incisal edge and the middle point of the cementoenamel junction were joined to depict the crown axis, and then the middle point of the cementoenamel junction with the root apex to depict the longitudinal axis were joined. The Collum angle was then measured. It is a supplement (180 degree $-\mathrm{x}$ ) of this value. A straight tooth will have a CA of zero degree, a lingually inclined root will have a positive angle, and a labially inclined root will have a negative angle. The traditional CA measurement, used in this study, is illustrated in Figure 1. The measurements were used to interpret the observed variations in the comparative study of the malocclusions. Gender variation of these values were also assessed. The results were then subjected to statistical analysis.

\section{Measurement of Labial Crown Root Angle}

The labial crown root angle (LCRA), is also constructed on a cephalometric radiograph with three points on the most anterior maxillary central incisor: the incisal edge (Incisal Superius; IS), the facial cemento enamel junction (fCEJ), and the apex of the incisor (UIA). The superius point is connected to the facial CEJ which depicts the crown axis. The apex of the incisor is joined with the facial CEJ which forms the root axis. The angle between this crown axis and root axis is measured. 
The LCRA is a supplement (180 degree $-\mathrm{x}$ ) of this value. The measurement of LCRA is depicted in figure 2 .

\section{Statistical Analysis}

The mean and standard deviation of for both the groups were calculated using Statistical Package for Social Science (SPSS). Independent $\mathrm{t}$-test was used to determine the significance between the groups. $\mathrm{P}$ value of $<0.05$ was considered significant.

\section{RESULTS}
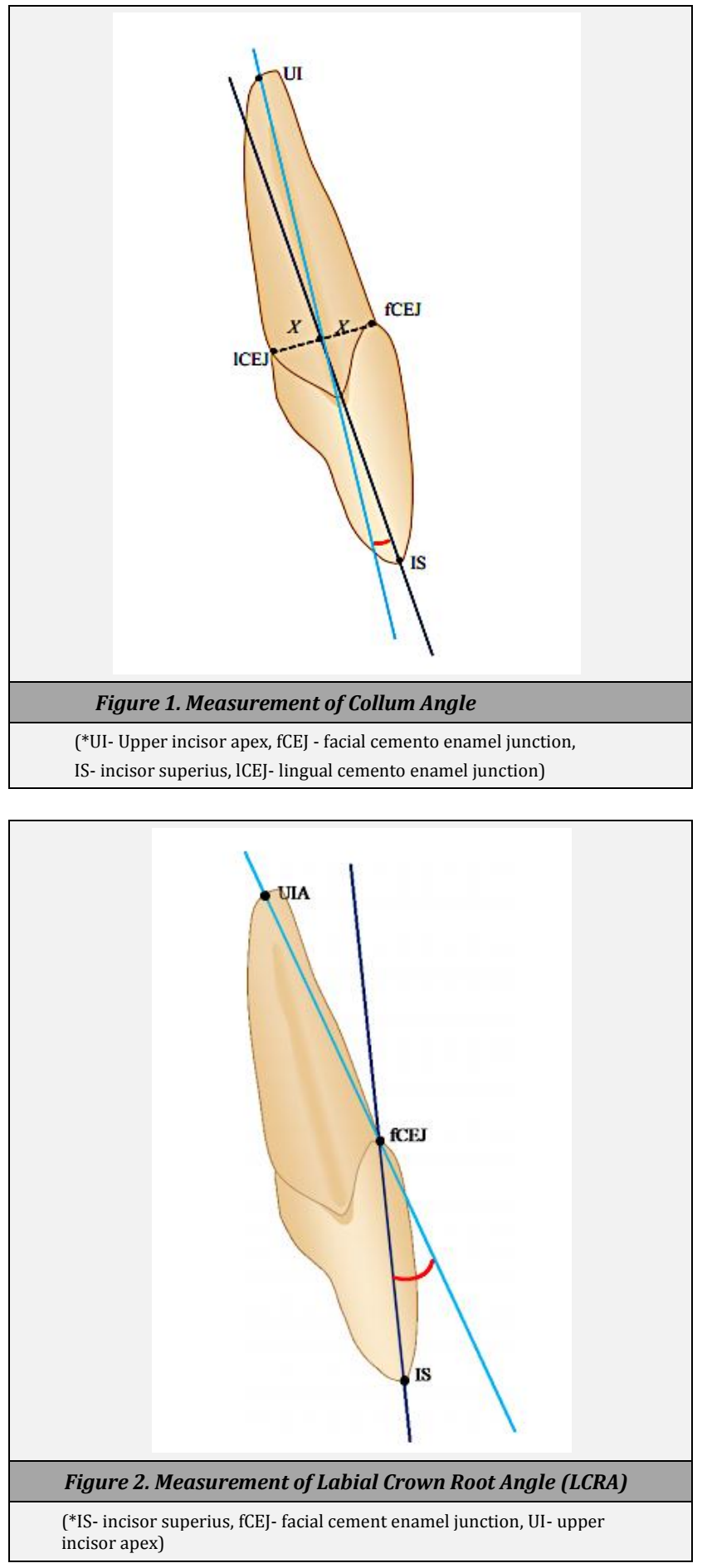
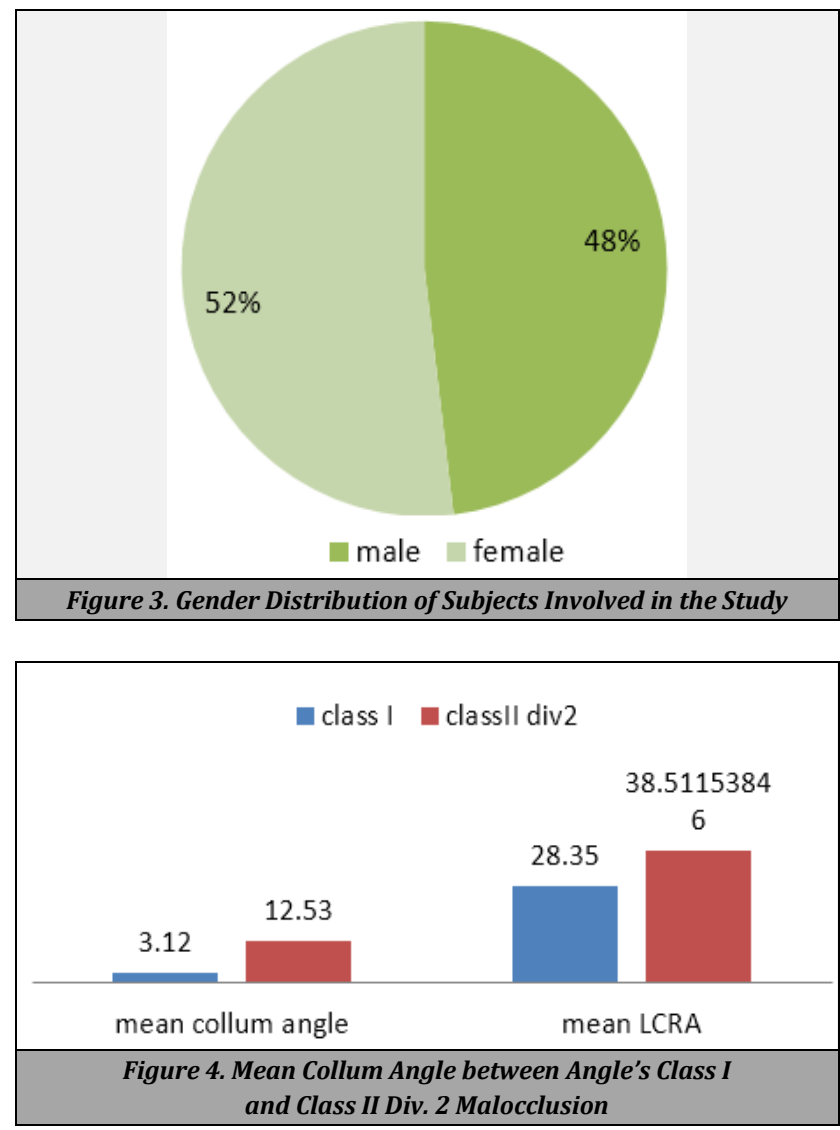

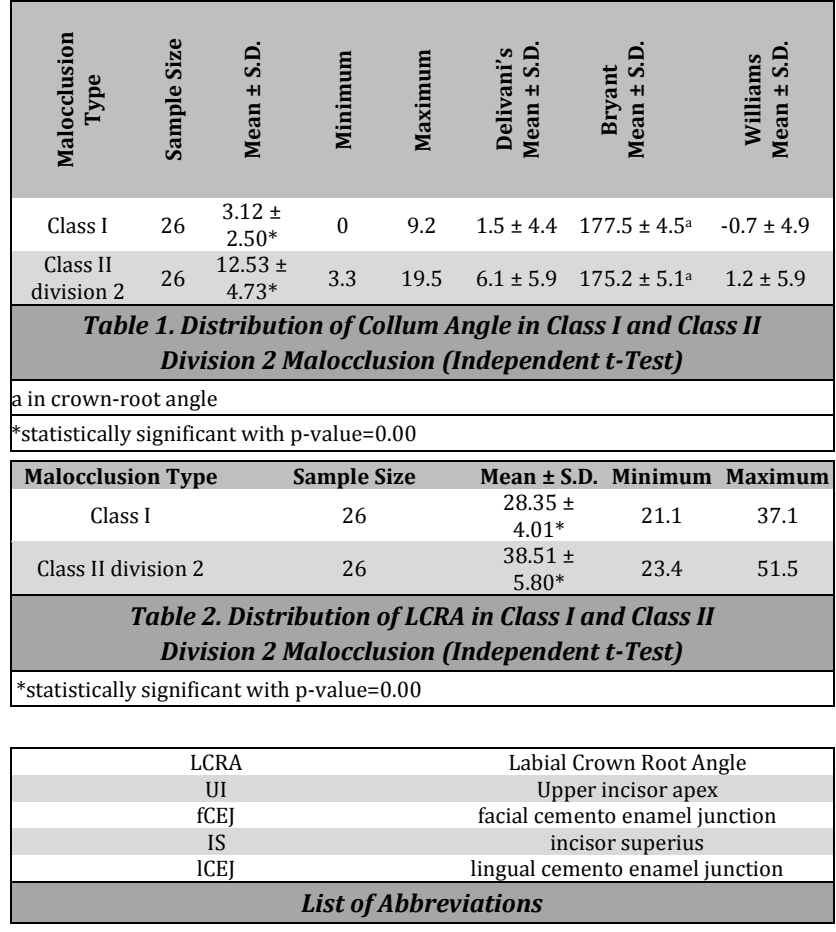

Both Class I and Class II division 2 groups had a sample size of 26 subjects each with a total sample size of 52. It included 25 male subjects and 27 female subjects. The ages ranged from 12 to 33 years, and the average age was 21 years. Ethical approval was obtained prior to the start of the study for Institutional Review Board of Saveetha University. The average value for Collum angle in Class I sample was $3.12 \pm$ $2.50^{\circ}$ with minimum being $0^{\circ}$ and maximum $9.2^{\circ}$. The average values for Class II division 2 was $12.53 \pm 4.73^{\circ}$ with minimum $3.3^{\circ}$ and a maximum $19.5^{\circ}$. (Table 1 ). 
The mean LCRA values for class I and class II division 2 malocclusion was found to be $28.35 \pm 4.01$ and $38.51 \pm 5.80$ respectively, with their maximum and minimum values being 21.1 and 37.1 degrees in class I malocclusion and 23.4 and 51.5 for class II division 2 malocclusion. The mean LCRA for class I and class II division 2 malocclusion is described in table 2 . Gender distribution of the study participants is depicted in figure 3.

Figure 4 is a line graph representing the distribution of collum angle between the two groups. This plot clearly shows that the collum angle is greater in Class II division 2 malocclusion. Independent t-test showed that there is a difference in the collum angle between class I and class II division 2 malocclusion, which is statistically significant. The difference in LCRA among the malocclusion types were also statistically significant (Table 2). The comparison between the two measurements and the difference in their values were also significant.

\section{DISCUSSION}

A total of 56 cephalograms were collected for this study of which 4 were excluded based on the exclusion criteria. The current study indicates that there is a wide variation in the crown root morphology of the maxillary central incisors. There are several explanations for this variation. It was postulated by Backlund and Harris et al that the crown of the maxillary central incisor is bent during its development. In case of class II division 2, this is due to the forces emanating from the lower lip and in case of class III it is due to the lower incisor, resulting in a "bent tooth".[10,12]

Several scholars indicate that heredity is also a primary cause for bending of the tooth.[13] Furthermore, Lapatki proposed that the levels of the lip line and lip pressure are external causal factors of bending between the crown and root long axes in maxillary anterior teeth.[12]

The results of this study shows that the collum angle in Class II division 2 malocclusion group is significantly greater than the class I malocclusion group, which is similar to the studies conducted by Delivanis, Bryant and Williams. ${ }^{[5][8][14]}$

William et al and Srinivasan et al traced the maxillary central incisor of various malocclusions and found that the collum angles differs significantly between class II division 1 and class II division 2 malocclusions.[14][15] Srinivasan et al stated that this variation is probably due to change in the position of lower lip line. Bauer et al studied the collum angle in Class I and Class II division 2 malocclusion and showed a greater collum angle in class II division 2 cases.[1]

Shen et al also compared the collum angle of different types of malocclusion and concluded that the collum angle was greater in patients with class II division 2 malocclusion. $\mathrm{He}$ inferred this to be due to the influence of different hereditary genes between the racial groups.[16]

It has been thought that the crown root angle for a maxillary central incisor in any type of malocclusion was assumed to be zero. Later, studies were conducted to evaluate on this angle. The importance of narrowing down to a mean crown root angle could be due to the susceptibility that the incisor root is torqued into the palatal cortical plate, resulting in root resorption or dehiscence.

The phenomenon of root resorption remains incompletely understood. Although root resorption was found to occur to some extent in orthodontic patients, ${ }^{[17]}$ it has been reported to be more frequent and severe in maxillary central incisors whose roots have been torqued into the palatal cortex.[18],[19],[20] Even though root resorption does not affect the prognosis of orthodontic tooth movement, it must be certainly considered as a primary goal of orthodontic treatment, if possible. ${ }^{[21][22]}$

Heravi et al studied the effect of collum angle on various types of orthodontic forces and lip pressure applied to the teeth. They concluded that the periodontal ligament of the maxillary central incisor experiences more stress during retraction when the collum angle is large. The opposite happened when the collum angle was small. It was also found that teeth with larger collum angle experienced lower intrusion forces as compared to ones with smaller collum angles.

The values obtained in the present study were in accordance to the results of the studies conducted by various authors as mentioned above.[23]

It was put forth by Bauer et al, that LCRA is clinically more useful and simpler than the collum angle. This was because the facial CEJ and the incisor superius, the anatomic points of the angle are typically identified in the cephalogram.[1]The ultimate goal of LCRA measurement is to describe the crownroot angulation. And the need for this study was to correlate these values with the collum angle.[1] The mean LCRA of class II division 2 was also found to be greater than class I malocclusion as in collum angle.

With the advent of CBCT (Cone Beam Computed Tomography), it is possible to carry out this study in a more accurate method for every tooth using analogue measurements. It is impractical to describe these landmarks and angles for every clinical case. Establishing mean values for both normal occlusion and other malocclusions like Angle's Class II and Class III would allow for proper prediction of incisor position within the alveolar cortex. This will also aid in proper appliance selection.[24]

This study has several limitations. One is being errors in the Facad software which is considered to be very small and negligible. The sample size for the study was set as 52 , which was again based on convenient sampling. Though the primary objective of this study was to measure the mean collum angle, the shape of the incisor should have been analysed. The variation in the crown shape, morphology and the plane of anterior palatal cortex would also likely contribute to the variation in crown-root angulation.

\section{CONCLUSIONS}

The mean collum angle of Class II division 2 malocclusion is different from zero and is significantly greater than Class I malocclusion. Within the limitations of this study, it can be concluded that the variation in crown root angulation is more in class II division 2 cases and is statistically significant. Also, 
Labial Crown Root Angle (LCRA), is a simple and useful measure to describe the crown-root angulation, which shows a statistically significant difference between class I and class II div 2 malocclusion. LCRA can be used as an adjunct to collum angle.

Financial or Other Competing Interests: None.

\section{REFERENCES}

[1] Bauer TJ. Maxillary central incisor crown-root relationships in class I normal occlusions and Class II division 2 malocclusions. MS (Master of Science) thesis, University of Iowa 2014. DOI: http://ir.uiowa.edu/etd/4572

[2] Kilpelainen P, Phillips C, Tulloch JF. Anterior tooth position and motivation for early treatment. Angle Orthod 1993;63(3):171-4.

[3] Mehu M, Dunbar R. Naturalistic observations of smiling and laughter in human group interactions. Behaviour 2008;145:1747-80.

[4] Proffit WR. The soft tissue paradigm in orthodontic diagnosis and treatment planning: a new view for a new century. J Esthet Dent 2000;12(1):46-49.

[5] Bryant RM, Sadowsky PL, Hazelrig JB. Variability in three morphologic features of the permanent maxillary central incisor. Am J Orthod 1984;86(1):25-32.

[6] Andrews LF. Straight wire: the concept and appliance. San Diego, CA: L.A. Wells Co 1989.

[7] Bryant RM, Sadowsky PL, Hazelrig JB. Variability in three morphologic features of the permanent maxillary central incisor. Am J Orthod 1984;86(1):25-32.

[8] Delivanis HP, Kuftinec MM. Variation in morphology of the maxillary central incisors found in class II, division 2 malocclusions. Am J Orthod 1980;78(4):438-43.

[9] Shailaja AM, Gowda NC, Gowda S. The collum angle of maxillary central incisors in different skeletal malocclusions - a cephaometric study. International Journal of Applied Dental Sciences 2016;2(3):33-6.

[10] Backlund E. Overbite and the incisor angle. Tram Eur Orthod Sot 1958:277-86.
[11] Rakosi T. An atlas and manual of cephalometric radiography. Philadelphia: Lea \& Febiger 1982.

[12] Harris EF, Hassankiadeh S, Harris JT. Maxillary incisor crown-root relationships in different angle malocclusions. Am J Orthod Dentofacial Orthop 1993;103(1):48-53.

[13] Logan WR. Deckbiss da clinical evaluation. Trans Eur Orthod Soc 1959;35:313e7.

[14] Williams A, Woodhouse C. The crown to root angle of maxillary central incisors in different incisal classes. $\mathrm{Br} \mathrm{J}$ Orthod 1983;10(3):159-61.

[15] Srinivasan B, Kailasam V, Chitharanjan A, et al. Relationship between crown-root angulation (collum angle) of maxillary central incisors in class II division 2 malocclusion and lower lip line. Orthodontics (Chic.) 2013;14(1):e66-74.

[16] Shen YW, Hsu JT, Wang HU, et al. The collum angle of the maxillary central incisors in patients with different types of malocclusion. J Dent Sci 2012;7(1):72-6.

[17] DeShields RW. A study of root resorption in treated class II division I malocclusions. Angle Orthod 1969;39(4):23145.

[18] Hoeve AT, Mulie RM. The effect of antero-posterior incisor repositioning on the palatal cortex as studied with laminography. J Clin Orthod 1976;10(11):804-22.

[19] Goldson L, Henrikson CO. Root resorption during Begg treatment: a longitudinal roentgenologic study. Am J Orthod 1975;68(1):55-66.

[20] Hickham JH. Directional force revisited. J Clin Orthod 1986;20(9):626-37.

[21] Ganesh ML, Pandiyan KS. Acceleration of tooth movement during orthodontic treatment-a frontier in orthodontics. J Pharm Sci \& Res 2017;9(5):741-4.

[22] Sree Vidhya TM, Navaneethan R. Root resorption with orthodontic intrusion review. JMSCR 2016;4(8):1194448.

[23] Heravi F, Salari S, Tanbakuchi B, et al. Effects of crownroot angle on stress distribution in the maxillary central incisors' PDL during application of intrusive and retraction forces: a three-dimensional fiite element analysis. Prog Orthod 2013;14:26.

[24] Lapatki BG, Mager AS, Schulte-Moenting J, et al. The importance of the level of the lip line and resting lip pressure in class II, division 2 malocclusion. J Dent Res 2002;81(5):323-8. 